\title{
Acupuncture in addition to budesonide does not further improve RQLQ apart from the sleep domain*
}

\section{Shan-ze Wang1, Ji-ping Zhao', Sheng-nan Guo², Shu-han Qu³, Wei-mei Zeng', Qi Xie', Yi Zhang', Sheng Chen'}

'Department of Acupuncture and Moxibustion, Dongzhimen hospital Affiliated to Beijing University of Chinese Medicine, Beijing, China

2 Department of Acupuncture and Moxibustion, Beijing Gulou Hospital of Traditional Chinese Medicine, Beijing, China

${ }^{3}$ Institute of Acupuncture and Moxibustion, China Academy of Chinese Medical Sciences, Beijing, China

${ }^{4}$ Department of Otolaryngology-Head and Neck Surgery, Beijing Chaoyang Hospital, Capital Medical University, Beijing, China
Rhinology Online, Vol 3: 184 - 192, 2020

http://doi.org/10.4193/RHINOL/20.053

*Received for publication:

June 30, 2020

Accepted: November 6, 2020

Published: November 20, 2020

\begin{abstract}
Background: Acupuncture can effectively alleviate the nasal symptoms of allergic rhinitis (AR); however, the mechanism is unclear. Previous studies suggested it may be related to improve hypothalamus-pituitary-adrenal (HPA) axis dysfunction.
\end{abstract}

Materials and Methods: The study was a randomised controlled trial including ninety patients with moderate to severe persistent AR. Subjects were randomised 1:1:1 to the 8-weeks treatment with mind-regulating acupuncture combined with intranasal glucocorticosteroids (INGC), conventional acupuncture combined with INGC and INGC alone (MA, CA, GC respectively). The primary outcome was the change from baseline in the Visual Analogue Scale (VAS) score of nasal symptoms. Rhinoconjunctivitis Quality of Life Questionnaire (RQLQ) score was a key secondary outcome. The levels of corticotrophin releasing hormone (CRH), cortisol (COR) and adrenocorticotropic hormone (ACTH) were also analysed.

Results: MA, CA and GC reduced VAS score and total RQLQ by a similar degree, but the sleep domain of RQLQ significantly improved in MA and CA compared to GC. Meanwhile, the COR and ACTH level increased, and CRH level decreased after treatment in MA and $C A$, but the changes showed no difference among the three groups after Bonferroni correction.

Conclusions: Although acupuncture combined with INGC may have potential effect on sleep quality, the combination therapy cannot further improve nasal symptoms or RQLQ compared to INGC alone in AR patients. The influence of acupuncture on HPA axis needs to be further explored.

Key words: acupuncture, allergic rhinitis, HPA axis, Intranasal glucocorticosteroids, RQLQ

\section{Introduction}

Acupuncture has been used to treat allergic rhinitis (AR) in China for a long time and now promoted worldwide ${ }^{(1)}$. The clinical evidence of the acupuncture's value in the treatment of AR was controversial. Although some of the studies failed to confirm the efficacy of acupuncture for $A R^{(2-4)}$, many studies reported positive results ${ }^{(5-9)}$ in recent years and a systematic review concluded that acupuncture might be safe and effective treatment for AR (10). Clinical practice guideline in AR from the American Academy of Otolaryngology-Head and Neck Surgery (AAO-HNS) also recommended acupuncture for AR patients who are interested in nonpharmacologic therapy ${ }^{(11)}$.

The protocol of acupuncture treatment in China varies in the selection of acupoints and the operation ${ }^{(7)}$. Since most AR patients have negative emotional states and sleep disturbances ${ }^{(8)}$, conventional acupoints may not satisfy the clinical needs. In traditional Chinese medicine (TCM) theory, mood disorders and sleep disturbance belong to the "mind disease" interacting with the primary disease which is similar to the concept "Psychosomatic disorder in western medicine". Therefore, acupuncture doctors usually choose the mind-regulated acupoints in addition to conventional acupoints for AR patients to improve the overall 
quality of life ${ }^{(9)}$. High quality clinical research is needed to access the effect of "mind-regulating" acupuncture and conventional acupuncture for AR.

Pieces of substantial evidences indicate that neuroendocrine immune system (NEI) is involved in the pathological mechanism of allergic diseases ${ }^{(15,16)}$. Studies have found that acupuncture improved the symptoms of AR by regulating the NEI system to produce anti-inflammatory effect ${ }^{(17,18)}$. HPA, an important part of the human neuroendocrine network was found to be activated by allergens in patients with rhinitis ${ }^{(19)}$. Moreover, the slow response of HPA axis positively correlated with the severity of symptoms in AR patients during the allergic season ${ }^{(20)}$. Therefore, we hypothesized that HPA axis dysfunction in AR patients might be caused by the chronic stress from the dual effects of physiological and psychological factors, and could result in recurrent AR attacks and difficult-to-control symptoms. Acupuncture can modulate the function of HPA axis bidirectionally (17), which might be one of the mechanisms in the treatment of AR. In clinical practice, many Chinese patients receive standard medication treatment and acupuncture treatment concurrently; however, there is no clinical study to evaluate the efficacy of this combination therapy until now. Therefore, we conducted a study on different acupuncture schemes combined with intranasal glucocorticosteroids (INGC) compared with INGC alone to evaluate the clinical value of acupuncture in the treatment of $A R$, and focused on the correlation between the intervention and the hormone level of HPA axis.

\section{Materials and methods}

Ethics

This study received ethics approval from the Institutional Review Boards of Beijing University of Chinese Medicine (Approval number: BZYYYDX-LL-20150208) and written informed consent was obtained from every participant.

\section{Trial design}

This was a prospective, randomized-controlled, evaluator-blinded study performed at the Department of Acupuncture and the Department of otolaryngology, Dongzhimen hospital Affiliated to Beijing University of Chinese Medicine, and the Department of otolaryngology, Beijing Chaoyang Hospital, between January 2017 and June 2018. The protocol of the study has registered in Acupuncture-Moxibustion Clinical Trial Registry in 2016 (ChiCTR-IOR-16009082, AMCTR-IOR-16000009. 22 August, 2016) and published in Trials ${ }^{(16)}$.

\section{Trial population}

\section{Inclusion criteria}

The trial population comprised adults 18 to 60 years of age with moderate-to-severe persistent allergic rhinitis (PAR), and healthy control subjects recruited 18 to 60 years old adults with no allergic disease and other chronic diseases to determine the normal level of HPA axis hormone. The moderate-to-severe PAR was diagnosed according to the criteria of Allergic Rhinitis and Its Impact on Asthma (ARIA) 2008 as following: the clinical history of persistent symptoms $>4$ days/week or $>4$ weeks/year, Mean Visual Analogue Scale (VAS) score of nasal symptoms $\geq 4$ for seven days during the screening period, and the evidence of at least one allergen tests ${ }^{(12)}$ : positive skin prick test (SPT) defined by a wheal diameter $\geq 3 \mathrm{~mm}$ and/or slgE $>0.35 \mathrm{kU} / \mathrm{L}$ to dust mite ${ }^{(22)}$.

\section{Exclusion criteria}

We excluded patients with 1) acute respiratory infection, acute sinusitis, chronic sinusitis, sinonasal lesions, or the history of nasal surgery; 2) paroxysmal respiratory diseases such as asthma; 3) Consumption of H1-antihistamines, steroids, anti-Recruitment histamine formulation, decongestant (applied to nasal cavity, oral cavity, or eyes), corticosteroids, antibiotics, and other medicines within 14 days; 4) TCM physiotherapy or other traditional medicine such as acupuncture, moxibustion, cupping within 14 days; 5) Women who are pregnant, lactating, or undergoing preparation; 6) Infectious diseases such as tuberculosis or hepatitis; 7) Smoking; 8) Scars at most selected acupoints; 9) individuals who are uncooperative during the treatments.

The data were excluded from analysis if subjects withdrew from the study or terminated by the investigators.

\section{Randomization and allocation concealment}

Randomisztion number were generated by an independent statistician with SAS 9.3.1 software (SAS Institute Inc., Cary, NC, USA) and the allocation was concealed in an opaque envelope. Only after an eligible participant provided informed consent and completed screening assessment, the researcher opened the envelope sequentially and the participant was then randomly assigned to MA (Mind-regulating acupuncture combined with INGC), CA (conventional acupuncture combined with INGC) and GC (only INGC) in a 1:1:1 ratio.

\section{Blinding}

Considering it was not feasible for blinding acupuncturists and patients, this study only blinded data researcher. The clinical data and laboratory data were collected through the electric notes to avoid communication with the participants by one data researcher who took no participant in the interventions. The data researcher was also responsible for statistical analysis and evaluation.

\section{Interventions}

The INGC applied equally in three groups. According to the stepwise medical treatment proposed by ARIA for moderate to severe PAR ${ }^{(17)}$, budesonide nasal spray (Rhinorcort, $64 \mu \mathrm{g}$ per 


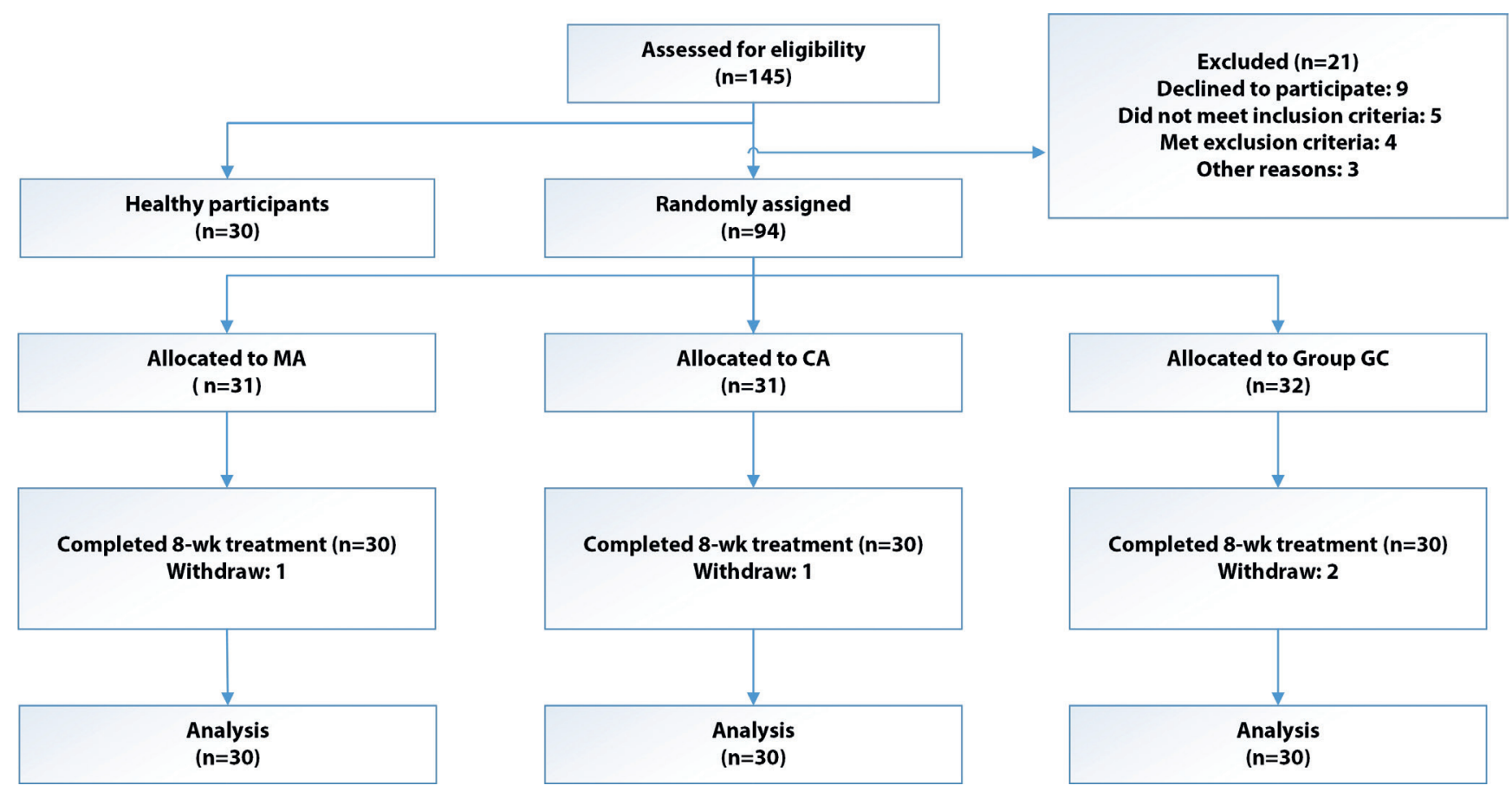

Figure 1. Overall trail design. $M A=$ Mind-regulating acupuncture combined with INGC; $C A=$ Conventional acupuncture combined with INGC; GC = INGC.

spray, AstraZeneca AB, Cambridge, UK) was provided at one puff per nostril twice a day in the first four weeks e (total $256 \mu \mathrm{g}$ budesonide per day). Then, budesonide nasal spray decreased to one puff per nostril in the morning for another four weeks after reassessed by the otolaryngologist

MA applied the mind-regulating acupuncture treatment combined with INGC, in which the acupoints were summarized by the acupuncture clinical experts ${ }^{(9)}$. During each session, the subjects received acupuncture at six acupoints including Bai Hui (DU20), Yin Tang (EX-HN3), Ying Xiang (LI20), Tai Chong (LR3), He Gu (LI4), Zu San Li (ST36), in the supine position firstly; then acupuncture at five acupoints including Da Zhui (DU14), Fei Shu (BL13), Pi Shu (BL20), Gan Shu (BL18), Shen Shu (BL23) in the prone position. $C A$ adopted the conventional acupuncture treatment combined with INGC, in which the acupoints were recommended in the Clinical Practice Guideline for Allergic Rhinitis Treatment with Acupuncture ${ }^{(18)}$. The acupuncture applied at five acupoints including Yingxiang (LI 20), Yintang (GV 29), Fengchi (GB 20), Hegu (LI 4), and Zusanli (ST 36)with patients in the supine position. The positions of all acupoints were referred to the WHO Standard ${ }^{(19)}$. In both groups, the needle retention lasted for 20 min after the achievement of "Deqi", which is a term in acupuncture meaning an intact transmission from the periphery to the central nervous system ${ }^{(20)}$. To promising the consistency and repeatability of the acupuncture technique, the procedures were made by two registered acupuncturists with more than three years of practising experience. The sterile stainless-steel disposable acupuncture needles ( $0.25 \mathrm{~mm} \times 40 \mathrm{~mm}$; Andy) were used for acupuncture. Each patient in MA and CA received three sessions per week for eight weeks.

\section{Outcomes}

The primary outcome was to determine the change of the VAS score to evaluate the improvement of overall nasal symptoms in the past weeks ${ }^{(21)}$. Subjects were instructed on how to make VAS assessment before treatment, and at week 2, 4, 6, 8 during treatment. For each assessment, the subjects were asked to place a mark at a $10 \mathrm{~cm}$ long caliper-shaped figure, which was divided into ten equal points, and labelled with 0-10 from left to right. The higher scores indicated worse troublesome the subjects suffered from the AR symptoms.

The secondary outcome was to determine the impact of AR on the quality of life through the Rhinoconjunctivitis Quality of Life Questionnaire (RQLQ) before intervention (week 0) and after the intervention (at the end of week 8) ${ }^{(22)}$. The questionnaire was tested from 7 aspects, including daily activities, sleep, non-nose or eye symptoms, practical problems, nose symptoms, eye symptoms and emotions, with a total of 28 questions. The higher score reflected the worse life quality associated with AR. The total RQLQ score, sleep domain, daily life domain (the sum of non-nose or eye symptoms domain and practical problems domain), eye symptoms domain and emotion domain were analysed.

Laboratory data included the concentrations of the serum corticotrophin releasing hormone $(\mathrm{CRH})$, serum cortisol (COR), and plasma adrenocorticotropic hormone (ACTH) detected by 
Table 1. Demographics and baseline characteristics.

\begin{tabular}{|c|c|c|c|c|}
\hline & MA & CA & GC & $\mathbf{P}$ \\
\hline Age (years) & $45.10 \pm 13.06$ & $40.87 \pm 10.87$ & $38.79 \pm 10.96$ & 0.106 \\
\hline \multicolumn{5}{|l|}{ Gender, n (\%) } \\
\hline Male & $9(30.0 \%)$ & $17(56.7 \%)$ & $10(33.3 \%)$ & 0.071 \\
\hline Female & $21(70.0)$ & $13(43.3 \%)$ & $20(66.7 \%)$ & \\
\hline Course of disease (month) & $55.07 \pm 32.95$ & $60.90 \pm 34.19$ & $59.60 \pm 29.94$ & 0.766 \\
\hline VAS & $7.50 \pm 1.80$ & $7.33 \pm 1.92$ & $7.07 \pm 2.24$ & 0.698 \\
\hline Total RQLQ score & $87.73 \pm 35.35$ & $90.47 \pm 29.55$ & $87.93 \pm 33.65$ & 0.938 \\
\hline Sleep domain & $8.63 \pm 4.90$ & $9.30 \pm 5.17$ & $7.87 \pm 5.06$ & 0.547 \\
\hline Daily life domain & $32.60 \pm 14.15$ & $35.47 \pm 15.06$ & $32.87 \pm 13.82$ & 0.695 \\
\hline Eye symptoms domain & $9.77 \pm 6.32$ & $10.23 \pm 7.51$ & $9.40 \pm 5.82$ & 0.887 \\
\hline Emotion domain & $12.50 \pm 7.17$ & $13.03 \pm 5.98$ & $11.93 \pm 6.99$ & 0.819 \\
\hline COR & $8.96 \pm 1.71$ & $8.92 \pm 1.55$ & $9.18 \pm 1.98$ & 0.827 \\
\hline ACTH & $104.22 \pm 36.10$ & $102.44 \pm 30.73$ & $105.55 \pm 46.46$ & 0.952 \\
\hline $\mathrm{CRH}$ & $27.93 \pm 7.63$ & $31.41 \pm 7.44$ & $27.54 \pm 7.22$ & 0.091 \\
\hline
\end{tabular}

$M A=$ Mind-regulating acupuncture combined with intranasal glucocorticosteroids (INGC); CA = Conventional acupuncture combined with INGC; GC $=$ INGC. RQLQ = Rhinoconjunctivitis Quality of Life Questionnaire; $\mathrm{CRH}=$ corticotrophin releasing hormone; COR = cortisol; $\mathrm{ACTH}=$ adrenocorticotropic hormone.

radioimmunoassay with GC-2010 Immune Counter (Thermo Fisher Scientific Company). Blood samples were collected in the fixed time (from 7:00 to 9:00 in the morning) before intervention (week 0) and after the intervention (at the end of week 8). The testing reagent manufacturer was Tianjin Jiuding Medical Bioengineering Co., Ltd. of China.

\section{Statistical methods}

\section{Sample size}

The study was an exploratory research. As stipulated in the Provisions for Drug Registration of China, the sample size for exploratory trials is 20-30 per group ${ }^{(23)}$. We aimed to recruit at least 30 cases for each group and healthy subjects.

Normality of data distribution was assessed by QQ-plots and Shapiro-Wilks test. The comparison of baseline characteristics among three groups was used one-way analysis of variance (ANOVA) for continuous variables and chi-square test for categorical variables. The VAS score of overall nasal symptoms was assessed by repeated measurement analysis of variance. A post-hoc power analysis was conducted in Power Analysis and Sample Size software (PASS 15; NCSS, USA). The comparison of secondary outcome and laboratory data within group was performed using Wilcoxon signed rank sum test, and the comparison among groups was performed by Kruskal-Wallis test followed by Bonferroni-corrected pairwise comparisons. Frequencies of occurrence of adverse events used Fisher 's exact test to assess. All data were calculated using a two-sided test. $P$ values less than 0.05 with corresponding confidence intervals of $95 \%$ were considered significant. Since five tests were performed in RQLQ scores and six tests were performed in laboratory data, the alpha level was adjusted for multiple comparisons using Bonferroni method. Thus, the results were considered significant when $P<0.01(0.05 / 5)$ in RQLQ scores and $P<0.0083(0.05 / 6)$ in laboratory data. All statistical analysis was performed using SPSS 23.0 software (IBM SPSS Statistics).

\section{Results}

Participants

A total of 94 patients were eligible and randomized for this study. At the same time, 30 healthy subjects $(H)$ who met the criteria were recruited. Four AR subjects dropped off due to patients' reasons inconvenient to continue the treatment, with one case dropped off in MA, one case in CA, and two cases in GC. Therefore, 30 AR subjects per group were analyzed finally. Figure 1 shows the trial design.

\section{Demographics and baseline data}

The demographics and baseline characteristics of the three groups were basically similar and comparable. There were no significant differences in the three groups of subjects in terms of age, gender, course of disease, family history, VAS score, RQLQ score and the concentration of HPA axis hormone (Table 1 Demographics and Baseline characteristics). 
Table 2. Overall nasal symptoms VAS score.

\begin{tabular}{lccc} 
& MA & CA & GC \\
\hline Week 0 & $7.50 \pm 1.80$ & $7.33 \pm 1.92$ & $7.07 \pm 2.24$ \\
\hline Week 2 & $5.77 \pm 2.24^{*}$ & $5.07 \pm 2.40^{*}$ & $5.00 \pm 2.24^{*}$ \\
\hline Week 4 & $4.90 \pm 1.19$ & $4.33 \pm 2.280$ & $4.07 \pm 2.50$ \\
Week 6 & $4.03 \pm 2.30$ & $3.53 \pm 2.06$ & $3.90 \pm 2.07$ \\
Week 8 & $2.77 \pm 1.74^{*}$ & $3.00 \pm 2.13$ & $3.47 \pm 2.10$ \\
\hline Holistic analysis & Among groups & Within groups & $\begin{array}{c}\text { Interaction } \\
\text { effect }\end{array}$ \\
(F, P) & $0.368,0.693$ & 73.614, 『0.001 & $1.489,0.164$
\end{tabular}

The VAS score of overall nasal symptoms in different time points of either group (mean with SD). Repeated measurement analysis of variance. No statistical difference at the same time point among groups. *The difference was statistically significant compared with the previous measurement within group, $\mathrm{P}<0.05$. MA = Mind-regulating acupuncture combined with INGC; CA = Conventional acupuncture combined with INGC; GC = INGC.

\section{Overall nasal symptom}

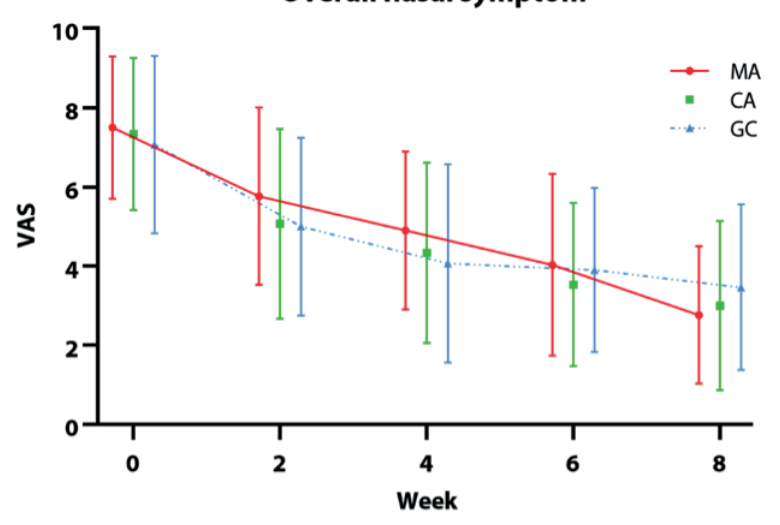

Figure 2. VAS score of overall nasal symptoms at different time points of either group (mean with SD). Repeated measurement analysis of variance. The treatment of each group has a statistically significant effect on the VAS scores (P凶0.001). No statistical difference at the same time point among groups. $M A=$ Mind-regulating acupuncture combined with INGC; CA = Conventional acupuncture combined with INGC; GC = INGC.

\section{Primary outcome}

The VAS scores of the overall nasal symptoms of each group roughly followed a normal distribution. The 8-weeks treatment significantly decreased the VAS scores from baseline in each group (MA: $4.73 \pm 2.01, C A: 4.33 \pm 2.70, G C: 3.60 \pm 2.27)$ [F (4, $84)=73.614, P<0.001]$. However, there was no statistically significant interaction between time and treatment measures $[F(8,170)=1.489, P=0.164]$. The VAS score has no statistical difference among groups at any time point of treatment $[F(2,87)$ $=0.368, P=0.693]$. A post-hoc power analysis showed a power of 0.9313 (Table 2 and Figure 2).

\section{Secondary outcome}

The corrected alpha level in the RQLQ scores was $0.01(0.05 / 5)$. The RQLQ total scores and sub-item scores of each group did not completely conform to the normal distribution. After eight weeks of treatment, the RQLQ total score $(P<0.001)$ and its sub-item scores $(P<0.01)$ were significantly improved in all three groups, except the sleep domain in group GC $(P=0.386)$. Comparing the change of total RQLQ score and each domain score between any two groups, there was only a significant difference on the sleep domain between MA with GC and CA with GC, but no difference between $M A$ and $C A$ (Corrected $P: P_{M A v s C}=0.002$, $P_{\text {CA vs GC }}=0.007, P_{\text {MAvs CA }}=1.000$; uncorrected P: $P_{\text {MA vs GC }}=0.001, P_{\text {CA vs }}$ $G_{G C}=0.002, P_{\text {MA vs CA }}=0.683$ ) (Figure 3).

The corrected alpha level in the laboratory data was 0.0083 (0.05/6). Hormones levels of each group did not completely conform to the normal distribution. Inter-group comparison of the change of COR from baseline was nonsignificant after Bonferroni correction (Corrected $\mathrm{P}: \mathrm{P}_{\text {MA v GC }}=0.016, \mathrm{P}_{\text {MA v CA }}=0.930, \mathrm{P}_{\text {CAvs }}$
${ }_{G C}=0.229$; uncorrected $P: P_{\text {MAvs GC }}=0.005, P_{\text {MAvs } C A}=0.076, P_{C A v s G C}$ $=0.310$ ). The change of ACTH and CRH from baseline showed no difference among three treatment groups either $\left(P_{A C T H}=0.121\right.$, $\left.P_{C R H}=0.119\right)$. However, we noticed that AR subjects usually had lower COR levels than healthy subjects at baseline before Bonferroni correction (Corrected P: $\mathrm{PM}_{\text {Avs H }}=0.044, \mathrm{P}_{\text {CAvs H }}=0.026$, $\mathrm{P}_{G C \text { vs H}}=0.070$; uncorrected $\mathrm{P}: \mathrm{P}_{\text {MAvs H }}=0.007, \mathrm{P}_{\text {CAvs H}}=0.004, \mathrm{P}_{G C \text { v H }}$ $=0.012$ ). Both MA and CA significantly increased COR levels after 8-weeks treatment $\left(P_{M A}<0.001, P_{C A}=0.011\right)$, and even similar to the healthy subjects' level at the end of interventions (Corrected $P: P_{\text {MAvs H }}=1.000, P_{\text {CAvs H }}=1.000$; uncorrected $\mathrm{P}: P_{\text {MAvs } H}=0.645, P_{C A}$ $\left.{ }_{v s} \mathrm{H}=0.729\right)$. No statistical difference was observed within $G C$ before and after treatment $\left(P_{G C}=0.797\right)$. Furthermore, the level of $\mathrm{CRH}$ and $\mathrm{ACTH}$ had no significant difference between the baseline of $A R$ subjects and healthy subjects $\left(P_{C R H}=0.086, P_{A C T H}\right.$ $=0.75)$. The ACTH level increased $\left(P_{M A}=0.035, P_{C A}=0.008\right)$ and $C R H$ level decreased $\left(P_{M A}=0.004, P_{C A}=0.033\right)$ in two acupuncture groups (Figure 4).

\section{Safety}

There was no serious adverse event occurred in the study. Two cases of bruising at Hegu (LI4) acupoint occurred in each of the two acupuncture treatment groups, and the bruising disappeared after 3 to 5 days, with no effect on the subsequent treatment. Eight cases reported nasal dryness which was related to INGC. There was no difference in occurrence frequencies of various adverse events among groups (Table 3 ).

\section{Discussion}

The combination of acupuncture with standardized medications 

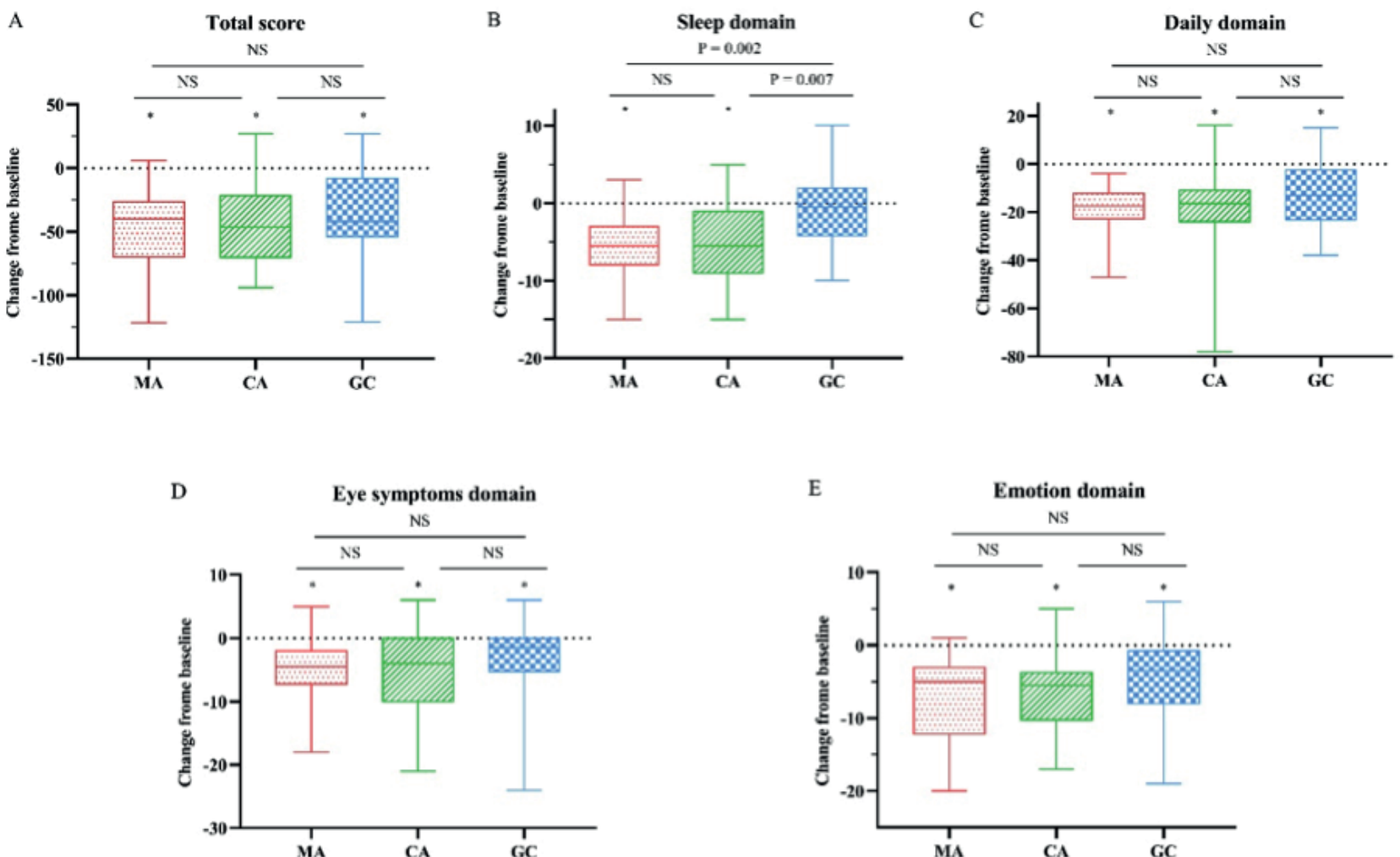

Figure 3. Change from baseline in subitem and total scores of Rhinoconjunctivitis Quality of Life Questionnaire (ROLQ) after 8-week treatment. Nonparametric test. The corrected alpha level was $0.01(0.05 / 5) .{ }^{*}$ The significant, difference within group before and after treatment. NS $=$ Not significant; MA = Mind-regulating acupuncture combined with INGC; CA = Conventional acupuncture combined with INGC; GC = INGC.

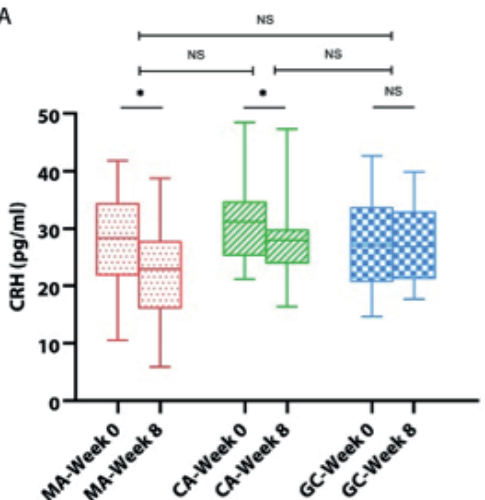

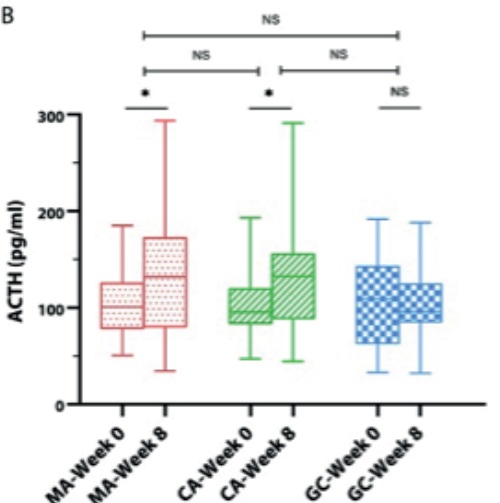

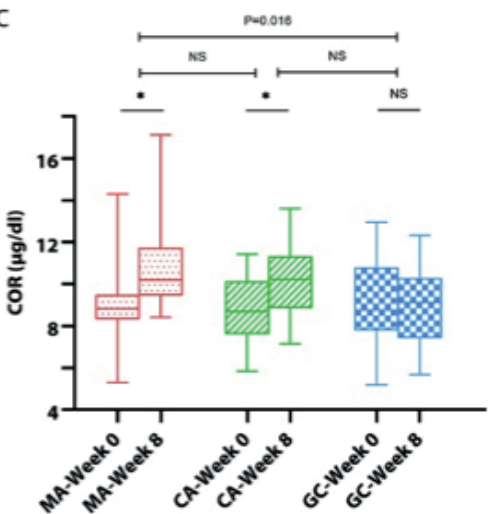

Figure 4. Concentration of serum corticotrophin releasing hormone (CRH), serum cortisol (COR), and plasma adrenocorticotropic hormone (ACTH). Nonparametric test. The corrected alpha level was 0.0083 (0.05/6). The change of COR from baseline between MA and GC had no significant difference after Bonferroni correction. ${ }^{*}$ The significant, difference within group before and after treatment. . NS = Not significant; MA = Mind-regulating acupuncture combined with INGC; CA = Conventional acupuncture combined with INGC; GC = INGC.

is common for AR management in China. This study explored the therapeutic efficacy of two different acupuncture schemes combined with INGC for AR. Previous acupuncture studies did not include consistent standard medication control. The efficacy of acupuncture was compared with loratadine in an RCT ${ }^{(29)}$, compared with the combination of cetirizine and budesonide nasal spray in a non-randomized controlled trial ${ }^{(30)}$, and both studies stated that acupuncture had the same efficacy as medication treatment without significance. Several studies showed the combination of INGC and other medications could further improve the efficacy ${ }^{(31-33)}$, so we tried to explore the effect of the INGC combined with acupuncture. 
Table 3. Adverse events.

\begin{tabular}{lcccc} 
& $\begin{array}{c}\text { MA } \\
(\mathbf{n}=\mathbf{3 0})\end{array}$ & $\begin{array}{c}\text { CA } \\
(\mathbf{n}=\mathbf{3 0})\end{array}$ & $\begin{array}{c}\mathbf{G C} \\
(\mathbf{n}=\mathbf{3 0})\end{array}$ & $\mathbf{P}$ \\
\hline Overall & 3 & 4 & 5 & 0.749 \\
Sever adverse events & 0 & 0 & 0 & - \\
Bruising & 2 & 2 & 0 & 0.351 \\
Nasal dryness & 1 & 2 & 5 & 0.168
\end{tabular}

The frequency of adverse events in each group was reported. Chi-square test. No significant difference in adverse events frequency among groups. $M A=$ Mind-regulating acupuncture combined with intranasal glucocorticosteroids (INGC); CA = Conventional acupuncture combined with INGC; GC = INGC.

Though two combined acupuncture schemes did not improve the nasal symptoms and overall quality of life scores compared with INGC alone, the sleep domain of RQLQ in the acupuncture groups significantly improved after treatment compared to GC group. Furthermore, sleep disorder is a common symptom in AR patients and affects their quality of life ${ }^{(34)}$. The sleep quality of patients should also be essentials for the evaluation of therapeutic efficacy, an additional treatment for insomnia may be required in AR patients to improve their life quality further ${ }^{(35,36)}$. The sleep disturbance is not only due to nasal symptoms, but also related to chronic systemic inflammation status ${ }^{(37)}$.The INGC may not enough for AR treatment from this perspective. In consistence with this study, several studies showed similar results that corticosteroid therapy did not significantly improve the RQLQ and Epworth Sleepiness Scale of AR patients ${ }^{(38,39)}$. Therefore, the effect of acupuncture combined with INGC on improving sleep quality is worth further study.

Previous studies have shown HPA axis dysfunction in allergic diseases. Some researches stated that the level of ACTH and COR elevated within 40 mins in patients with allergic rhinitis exposed to allergic substances ${ }^{(40)}$, and HPA axis responsiveness to stress was blunted in atopic patients ${ }^{(20,41,42)}$. We hypothesized that the HPA axis of moderate to severe AR patients would be in hypofunction due to long-term inflammatory stimulation. Therefore, we focused on the basic level of HPA axis related hormones. The present study showed that their COR level was lower than that of healthy participants, but the difference was non-significant after Bonferroni correction. Animal experiments showed that acupuncture significantly activate the inhibition status of HPA axis with elevating the level of ACTH and COR in the model rats with asthma ${ }^{(43)}$. However, our laboratory data cannot prove the impact of acupuncture on HPA related hormones. Although there is a trend that acupuncture enhanced the activity of HPA axis by increasing the low level of COR in AR patients to that of healthy subjects, possible differences observed in hormone changes between groups did not reach conventional levels of statistical significance after Bonferroni correction. The alpha level of 0.05 was artificial statistical standard, hence, no statistical significance after multiple correction cannot exclude the possibility of positive. The present study explored the influence of acupuncture on HPA axis and found interesting trend regulation, so further research is needed to determine the correlation in a larger sample size.

$A$ axis by increasing the low level of COR in AR patients to that of healthy subjects, though possible differences observed in $\mathrm{ACTH}$ or $\mathrm{CRH}$ between groups did not reach conventional levels of statistical significance. In contrast, INGC (budesonide) had no significant effect on COR level.

Interestingly, based on the theory of TCM, we expected that the mind-regulating acupuncture would have a better effect on the improvement of emotion and sleep quality of AR patients than conventional acupuncture. However, the study showed no significant difference in emotion or sleep quality between the two acupuncture groups, which indicated that the conventional acupuncture scheme also played a positive role in the improvement of sleep quality. Otherwise, the mind-regulating acupuncture might trigger more inclusive regulating effects on the mental area for AR patients, which have not been fully reflected through the questionnaire used in this study.

There were several limitations. Firstly, we have done a lot of testing on secondary outcomes, though the present study was an exploratory trial and Bonferroni correction was made in secondary outcomes. Secondly, there was possibly a ceiling effect caused by INGC that limited the improvement of symptoms of acupuncture. Alternatively, the research design of the personalized or on-demand INGC application may be more suitable to reflect the therapeutic value of acupuncture. Finally, some studies have pointed out that high-dose or long-term use of budesonide can inhibit the HPA axis ${ }^{(44,45)}$, and the other declared short-term use and conventional dosage of budesonide had no significant effect on the HPA axis ${ }^{(46)}$. It would have been better to use mometasone or fluticasone instead of budesonide in this study to exclude the suspicion of HPA inhibition related to budesonide.

\section{Conclusions}

Although acupuncture combined with INGC may have potential effect on sleep quality, the combination therapy cannot further improve nasal symptoms or RQLQ compared to INGC alone in AR patients. The regulation of acupuncture on HPA axis needs to be explored further.

\section{List of abbreviations}

AR: Allergic rhinitis; INGC: Intranasal glucocorticosteroids; TCM: Traditional Chinese medicine; HPA: Hypothalamus-pituitary- 
adrenal; NEI: Neuroendocrine immune system; VAS: Visual Analogue Scale; SPT: positive skin prick test; MA: Mind-regulating acupuncture combined with INGC; CA: Conventional acupuncture combined with INGC; GC: INGC alone; $\mathrm{H}$ : Healthy subjects; RQLQ: Rhinoconjunctivitis Quality of Life Questionnaire; ANOVA: Analysis of variance.

\section{Acknowledgments}

We gratefully appreciate the statistically guidance and assistance of professor Yan Liu from Dongzhimen hospital Affiliated to Beijing University of Chinese Medicine.

\section{Ethics approval and consent to participate}

Not applicable.

\section{Authorship contribution}

Study concept and design: SC, JPZ. Acupuncture treatment: SC, SHQ, WMZ. Otolaryngologist consultant: YZ. Acquisition, analysis, or interpretation of data: SZW, SNG. Drafting of the manuscript: S-Z Wang, S-H Qu, W-M Zeng. Obtaining of funding: SC, JPZ. Revision of the Manuscript: YZ, SZW, SC, SNG, QX. Study supervision: JPZ.

\section{Conflict of interest}

There was no commercial interest existing during the whole research process and the manuscript completing.

\section{Funding}

Project funded by the Natural Science Foundation of Beijing Municipality, China (grant number 7164272) and supported by National Key R \& D Projects (Grant number: 2019YFC1712200).

\section{Consent for publication}

Not applicable.

\section{Availability of data and materials}

Not applicable.

\section{References}

1. Wu AW, Gettelfinger JD, Ting JY, Mort C Higgins TS. Alternative therapies for sinusitis and rhinitis: a systematic review utilizing a modified Delphi method. Int Forum Allergy Rhinol 2020; 10:496-504.

2. MD S, RK G, SY L, et al. Clinical practice guideline: Allergic rhinitis. Otolaryngol Head Neck Surg 2015; 152:S1-43.

3. Wise SK, Lin SY, Toskala E. International consensus statement on allergy and rhinology: allergic rhinitis-executive summary. Int Forum Allergy Rhinol 2018; 8:85-107.

4. Adam D, Grabenhenrich L, Ortiz M, Binting S, Reinhold T, Brinkhaus B. Impact of acupuncture on antihistamine use in patients suffering seasonal allergic rhinitis: secondary analysis of results from a randomised controlled trial. Acupuncture Med 2018; 36:139-145

5. Witt CM, Reinhold T, Jena S, Brinkhaus B, Willich SN. Cost-effectiveness of acupuncture in women and men with allergic rhinitis: a randomized controlled study in usua care. Am J Epidemiol 2009; 169:562-571.

6. Brozek JL, Bousquet J, Baena-Cagnani CE, et al. Allergic Rhinitis and its Impact on Asthma (ARIA) guidelines: 2010 revision. J Allergy Clin Immunol 2010; 126:466-476.

7. Merrill SJ, and Minucci SB. Thyroid Autoimmunity: An Interplay of Factors. Vitamins and hormones 2018; 106:129-145.

8. Roxbury CR, Qiu M, Shargorodsky J, Lin SY. Association between allergic rhinitis and poor sleep parameters in U.S. adults. Int Forum Allergy Rhinol 2018; 8:1098-1106.

9. Sheng $C$, Jun W, Peng B, et al. Moderate and severe persistent allergic rhinitis treated with acupuncture: a randomized controlled trial. Chin Acupuncture Moxibustion 2015;

\section{5:1209-1213}

10. Faravelli C, Lo Sauro C, Lelli L, et al. The role of life events and HPA axis in anxiety disorders: a review. Curr Pharm Des 2012 18:5663-5674

11. Marshall GD, Jr., Agarwal SK. Stress, immune regulation, and immunity: applications for asthma. Allergy Asthma Proc 2000; 21:241246.

12. Kalogeromitros $D$, Syrigou EK, Makris M, et al. Nasal provocation of patients with allergic rhinitis and the hypothalamic-pituitaryadrenal axis. Ann Allergy Asthma Immunol. 2007; 98:269-273

13. McDonald JL, Smith PK, Smith CA, Changl Xue C, Golianu B, Cripps AW. Effect of acupuncture on house dust mite specific $\lg \mathrm{E}$, substance $\mathrm{P}$, and symptoms in persistent allergic rhinitis. Ann Allergy Asthma Immunol. 2016; 116:497-505.

14. Jung D, Lee S, Hong S. Effects of acupuncture and moxibustion in a mouse mode of allergic rhinitis. Otolaryngol Head Neck Surg. 2012; 146:19-25.

15. Ding SS, Hong SH, Wang C, Guo Y, Wang ZK $\mathrm{Xu}$ Y. Acupuncture modulates the neuroendocrine-immune network. QJMs 2014 107:341-345

16. Chen S, Qu SH, Zhang Y.et al. Impact of acupuncture for allergic rhinitis on the activity of the hypothalamus-pituitary-adrenal axis: study protocol for a randomized controlled trial. Trials 2019: 20:372.

17. Bousquet J, Khaltaev N, Cruz AA, et al Allergic Rhinitis and its Impact on Asthma (ARIA) 2008*. Allergy 2008; 63:8-160

18. Chen S, Guo SN, Marmori F, et al. Clinical Practice Guideline for Allergic Rhinitis Treatment with Acupuncture. Chin J Integr Med. 2020, doi: 10.1007/s11655-020-3161-0.
19. Organization WHO. WHO standard acupuncture point locations in the Western Pacific Region. 2008

20. Zhang Y, Zhang C, Yan M, Wang N, Liu J, Wu A. The effectiveness of PC6 acupuncture in the prevesntion of postoperative nausea and vomiting in children: A systematic review and meta-analysis. Paediatric anaesthesia 2020. 30(5):552-563.

21. Del Cuvillo A, Santos V, Montoro J, et al. Allergic rhinitis severity can be assessed using a visual analogue scale in mild, moderate and severe. Rhinology 2017; 55:34-38.

22. Dietz de Loos DA, Segboer CL, Gevorgyan A, Fokkens WJ. Disease-specific quality-oflife questionnaires in rhinitis and rhinosinusitis: review and evaluation. Curr Allergy Asthma Rep 2013; 13:162-170

23. State Administration for Market Regulation. Measures for the Administration of Drug Registration (Bureau Order No. 28). State Administration for Market Regulation (Ed), 2007.

24. Chen H, Zhang L, Lou H, Wang Y, Cao F, Wang $C$. A randomized trial of comparing a combination of montelukast and budesonide with budesonide in allergic rhinitis. Laryngoscope 2019

25. Gross GN, Berman G, Amar NJ, Caracta CF, Tantry SK. Efficacy and safety of olopatadine-mometasone combination nasal spray for the treatment of seasonal allergic rhinitis. Ann Allergy Asthma Immunol. 2019. 122:630-638.e633.

26. Wang SZ, Yao $Y$, Zhang $X J$, et al. Combination treatment of allergic rhinitis using ketotifen fumarate and budesonide administered as nasal sprays Int J Clin Pharmacol Ther 2020; 58:195-197.

27. Sastre J, Mosges R. Local and systemic safe- 
ty of intranasal corticosteroids. J Investig Allergol Clin Immunol 2012; 22:1-12.

28. Yanai K, Rogala B, Chugh K, Paraskakis E, Pampura AN, Boev R. Safety considerations in the management of allergic diseases: focus on antihistamines. Curr Med Res Opin 2012; 28:623-642

29. Glockler-Lauf SD, Finkelstein Y, Zhu J, Feldman LY, To T. Montelukast and Neuropsychiatric Events in Children with Asthma: A Nested Case-Control Study. J Pediatr 2019; 209:176-182.e174.

30. Choi SM, Park JE, Li SS, et al. A multicenter, randomized, controlled trial testing the effects of acupuncture on allergic rhinitis. Allergy 2013; 68:365-374.

31. Brinkhaus B, Ortiz $M$, Witt $C M$, et al. Acupuncture in patients with seasonal allergic rhinitis: a randomized trial. Ann Intern Med 2013; 158:225-234

32. Xue CC, Zhang AL, Zhang CS, DaCosta C, Story DF, Thien FC. Acupuncture for seasonal allergic rhinitis: a randomized controlled trial. \#N/A 2015; 115:317-324.e311.

33. Mi J, Chen X, Lin X, et al. Treatment of persistent allergic rhinitis via acupuncture at the sphenopalatine acupoint: a randomized controlled trial. Trials 2018; 19:28.

34. Chen $Y D$, Jin $X Q$, $Y u$ MH, Fang $Y$, Huang LQ Acupuncture for moderate to severe allergic rhinitis: A non-randomized controlled trial. Chin J Integr Med 2016; 22:518-524.

35. Liu J, Zhang $X$, Zhao Y, Wang Y. The association between allergic rhinitis and sleep: A systematic review and meta-analysis of observational studies. PLoS One 2020 15:e0228533.

36. Thompson A, Sardana N, Craig TJ. Sleep impairment and daytime sleepiness in patients with allergic rhinitis: the role of congestion and inflammation. Ann Allergy Asthma Immunol. 2013; 111:446-451.

37. Yamada $T$, Yamamoto $H$, Kubo $S$, et al. Efficacy of mometasone furoate nasal spray for nasal symptoms, quality of life, rhinitis-disturbed sleep, and nasal nitric oxide in patients with perennial allergic rhinitis. Allergy Asthma Proc 2012; 33:e9-16.

38. Meltzer EO, Munafo DA, Chung W, Gopalan G, Varghese ST. Intranasal mometasone furoate therapy for allergic rhinitis symptoms and rhinitis-disturbed sleep. Ann Allergy Asthma Immunol. 2010; 105:65-74.

39. Kalpaklioğlu AF, Kavut AB, Ekici M. Allergic and nonallergic rhinitis: the threat for obstructive sleep apnea. Ann Allergy Asthma Immunol. 2009; 103:20-25.

40. Ferrando $M$, Bagnasco D, Roustan $V_{\text {, }}$ Canonica GW, Braido F, Baiardini I. Sleep complaints and sleep breathing disorders in upper and lower obstructive lung diseases. J Thorac Dis 2016; 8:E716-725.

41. Lo Martire V, Caruso D, Palagini L, Zoccoli G, Bastianini S. Stress \& sleep: A relationship lasting a lifetime. Neurosci Biobehav Rev. 2019; S0149-7634(19)30149-6

42. Eshkevari L, Permaul E, Mulroney SE. Acupuncture blocks cold stress-induced increases in the hypothalamus-pituitaryadrenal axis in the rat. J Endocrinol 2013; 217:95-104.

43. Le JJ, Yi T, Qi L, Li J, Shao L, Dong JC. Electroacupuncture regulate hypothalamic-pituitary-adrenal axis and enhance hippocampal serotonin system in a rat model of depression. Neuroscience letters 2016; 615:66-71.

44. Zhu J, Chen Z, Meng $Z$, et al. Electroacupuncture Alleviates Surgical Trauma-Induced Hypothalamus Pituitary
Adrenal Axis Hyperactivity Via microRNA-142. Front Mol Neurosci. 2017; 10:308.

45. Wei Y, Dong M, Zhong L, et al. Regulation of hypothalamic-pituitary-adrenal axis activity and immunologic function contributed to the anti-inflammatory effect of acupuncture in the OVA-induced murine asthma model. Neuroscience letters 2017; 636:177183.

46. Buske-Kirschbaum A, Ebrecht M, Hellhammer DH. Blunted HPA axis responsiveness to stress in atopic patients is associated with the acuity and severeness of allergic inflammation. Brain Behav Immun. 2010; 24:1347-1353

47. Rajcani J, Solarikova P, Buzgoova K, Brezina I, Jezova D. Patients with atopy exhibit reduced cortisol awakening response but not cortisol concentrations during the rest of the day. Immunol Res 2019; 67:176-181.

48. Ratner PH, Miller SD, Hampel FC, Jr. Melchior A, Dunbar SA, Tantry SK. Oncedaily treatment with beclomethasone dipropionate nasal aerosol does not affect hypothalamic-pituitary-adrenal axis function. Ann Allergy Asthma Immunol. 2012; 109:336-341.

49. Kosoglou T, Cutler DL, Staudinger H, Herron $J M$. Once-daily evening dosing of mometasone furoate administered via a dry powder inhaler does not adversely affect the hypothalamic-pituitary-adrenal axis. Chest 2010; 137:115-121.

50. Ortiz $M$, Witt $C M$, Binting $S$, et al. A randomised multicentre trial of acupuncture in patients with seasonal allergic rhinitis--trial intervention including physician and treatment characteristics. BMC Complement Altern Med. 2014: 14:128.

\section{Dr. Sheng Chen \\ Department of Acupuncture and Moxi- \\ bustion \\ Dongzhimen hospital Affiliated to Bei- jing University of Chinese Medicine No.5 Haiyuncang Hutong 100700, Beijing \\ China}

Tel and Fax: +86-01063793828

E-mail: qdchensheng@126.com ORCID ID: 0000-0001-5457-0226

\section{Dr. Yi Zhang}

Department of Otolaryngology-Head and Neck Surgery

Beijing Chaoyang Hospital, Capital

Medical University

No.8 Gongtinanlu

100020, Beijing

China

Tel and Fax: +86-01085231350

E-mail: dr.zhangyi@hotmail.com ORCID ID: 0000-0002-6126-8014

ISSN: 2589-5613 / @2020 The Author(s). This work is licensed under a Creative Commons Attribution 4.0 International License. The images or other third party material in this article are included in the article's Creative Commons license, unless indicated otherwise in the credit line; if the material is not included under the Creative Commons license, users will need to obtain permission from the license holder to reproduce the material. To view a copy of this license, visit http://creativecommons.org/licenses/by/4.0/ 